\title{
Visualizing the Dynamics of Subsurface Defects with Time-Resolved Dark-Field X-ray Microscopy
}

\author{
Leora E Dresselhaus-Marais
}

Lawrence Livermore National Labs

ldresselhauscooper@alum.mit.edu

A material responds to its surroundings via residual changes in its structure that change its corresponding properties. The macroscopic structural evolution is instigated by the dynamics of statistical populations of defects that move, interact, and pattern - causing atomicscale defects to create 3D networks of boundaries that comprise the heterogeneous "real-world" materials. While techniques exist to probe material defects, they are mainly limited to surface measurements or rastered scans that cannot measure the dynamics of irreversible or stochastic processes characteristic of defect dynamics. In this talk, I will introduce time-resolved dark-field X-ray microscopy (tr-DFXM) as a new tool to capture movies that visualize dislocation dynamics in single- and poly-crystals at the mesoscale. I will start by describing the infrastructure we have developed to build and align the microscope, then to interpret and quantify the information captured in our movies. With this new tool, I will then demonstrate how dislocation patterns evolve at high temperatures in aluminum (Fig. 1). Tr-DFXM holds important opportunities for future studies on mesoscale dynamics, as it can inform models that have previously been refined only by indirect measurements and multi-scale models.

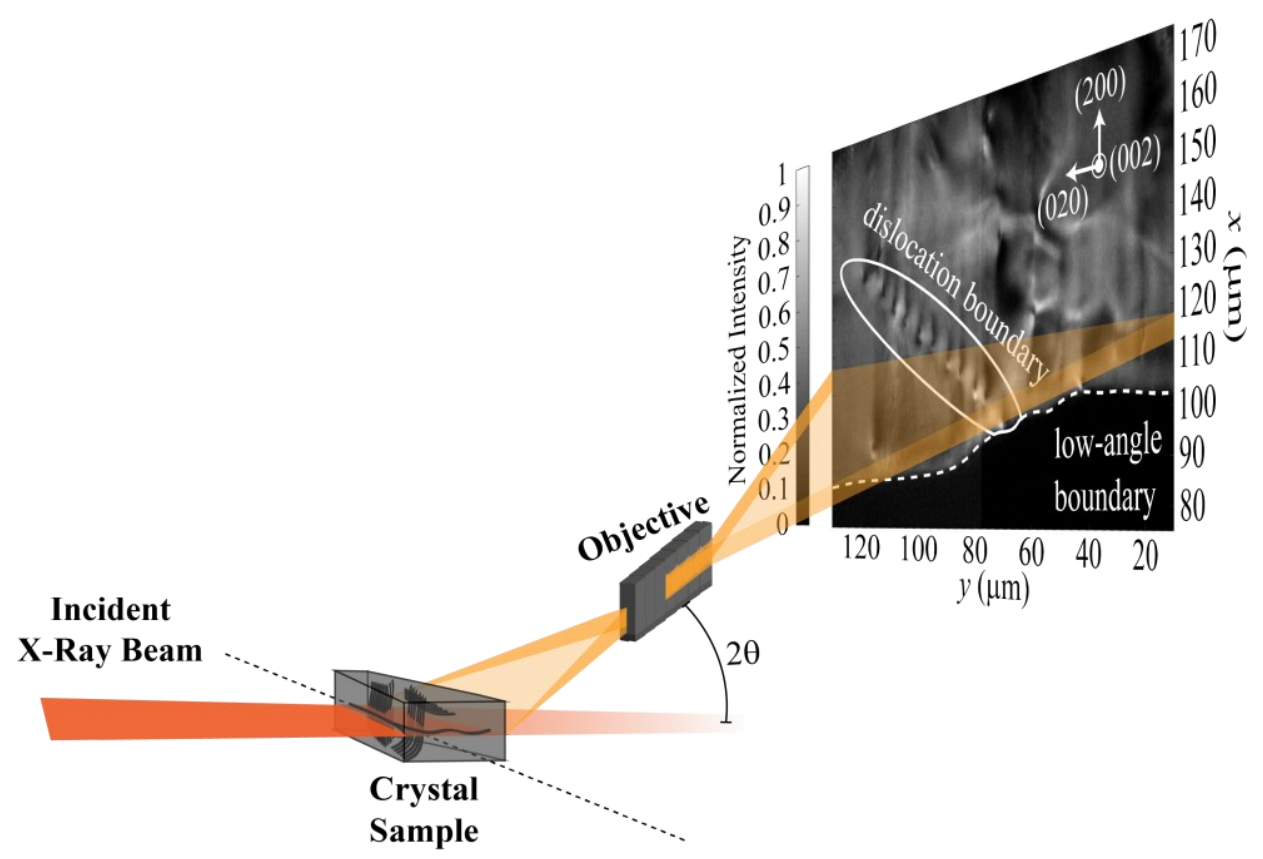

Figure 1: Schematic of the tr-DFXM setup used to collect dislocation movies in single-crystal aluminum at high temperatures.

This work was performed in part under the auspices of the U.S. Department of Energy by Lawrence Livermore National Laboratory under Contract DE-AC52-07NA27344. 